\title{
BMJ Open Can interprofessional teamwork reduce patient throughput times? A longitudinal single-centre study of three different triage processes at a Swedish emergency department
}

Jenny Liu, Italo Masiello, Sari Ponzer, Nasim Farrokhnia

To cite: Liu J, Masiello I,

Ponzer S, et al. Can interprofessional teamwork reduce patient throughput times? A longitudinal single-centre study of three different triage processes at a Swedish emergency department. BMJ Open 2018;8:e019744. doi:10.1136/ bmjopen-2017-019744

- Prepublication history for this paper is available online. To view these files, please visit the journal online (http://dx.doi. org/10.1136/bmjopen-2017019744).

Received 22 September 2017 Revised 8 March 2018 Accepted 14 March 2018
Check for updates

Department of Clinical Science and Education, Karolinska Institutet, Södersjukhuset, Stockholm, Sweden

Correspondence to Dr Jenny Liu; jenny.liu@ki.se

\section{ABSTRACT}

Objective To determine the impact on emergency department (ED) throughput times and proportion of patients who leave without being seen by a physician (LWBS) of two triage interventions, where comprehensive nurse-led triage was first replaced by senior physician-led triage and then by interprofessional teamwork.

Design Single-centre before-and-after study.

Setting Adult ED of a Swedish urban hospital.

Participants Patients arriving on weekdays 08:00 to 21:00 during three 1-year periods in the interval May 2012 to November 2015. A total of 185806 arrivals were included.

Interventions Senior physicians replaced triage nurses May 2013 to May 2014. Interprofessional teamwork replaced the triage process on weekdays 08:00 to 21:00 November 2014 to November 2015.

Main outcome measures Primary outcomes were the median time to physician (TTP) and the median length of stay (LOS). Secondary outcome was the LWBS rate.

Results The crude median LOS was shortest for teamwork, $228 \mathrm{~min}(95 \% \mathrm{Cl} 226.4$ to 230.5 ) compared with $232 \mathrm{~min}$ ( $95 \% \mathrm{Cl} 230.8$ to 233.9 ) for nurse-led and $250 \mathrm{~min}(95 \% \mathrm{Cl} 248.5$ to 252.6$)$ for physician-led triage. The adjusted LOS for the teamwork period was 16 min shorter than for nurse-led triage and 23 min shorter than for physician-led triage. The median TTP was shortest for physician-led triage, $56 \mathrm{~min}(95 \% \mathrm{Cl} 54.5$ to 56.6$)$ compared with $116 \mathrm{~min}(95 \% \mathrm{Cl} 114.4$ to 117.5$)$ for nurseled triage and $74 \mathrm{~min}(95 \% \mathrm{Cl} 72.7$ to 74.8$)$ for teamwork. The LWBS rate was $1.9 \%$ for nurse-led triage, $1.2 \%$ for physician-led triage and $3.2 \%$ for teamwork. All outcome measure differences had two-tailed $p$ values $<0.01$.

Conclusions Interprofessional teamwork had the shortest length of stay, a shorter time to physician than nurse-led triage, but a higher LWBS rate. Interprofessional teamwork may be a useful approach to reducing ED throughput times.

\section{INTRODUCTION}

Emergency department (ED) crowding is a growing problem worldwide. ${ }^{1-5}$ Patients risk suffering prolonged pain, inconvenience
Strengths and limitations of this study

- Two interventions are analysed and three triage processes compared in the same emergency department (ED).

- The large study population allows an accurate comparison of the triage processes.

- The control and study periods of 1 year each compensate for seasonal fluctuations and allow each intervention to stabilise after the initial implementation.

- The results from a large urban ED may not be generalisable to other ED settings.

- The-before-and-after design may not claim a causality between the interventions and the outcomes, although no other changes took place during the study period.

and poor outcomes due to delays in emergency care. ${ }^{26-8} \mathrm{ED}$ crowding can also lead to dissatisfaction among staff and a high rate of turnover as well as increased aggression and violence from frustrated patients. ${ }^{9-11}$ Many external factors can contribute to ED crowding, such as an increasing patient volume, increased complexity and acuity of patients' diseases, and a lack of beds for patients admitted from the ED into the hospital's other wards or departments. ${ }^{126}$

In the 1950s, triage of patients became a key strategy to handle the crowding problem. $^{12}$ The objective of an ED triage process is to quickly sort patients according to their priority of care. A quick triage check is typically performed by a nurse and consists of a simple visual assessment of the patient's medical urgency. More comprehensive triage systems, also typically carried out by a nurse, involve taking vital signs and patient history before the priority of care is determined. ${ }^{13}$ In nurse-led triage, the protocol may also allow nurses to order laboratory tests and 
radiographs. ${ }^{12}$ Comprehensive nurse-led triage using different standardised acuity protocols has been widely implemented since the 1990s. However, the evidence of its reliability and validity is scarce. ${ }^{121415}$

During the last two decades, some EDs have introduced physicians in the triage process to improve throughput and patient flow. These interventions have been reported to result in a reduced waiting time to physician assessment, fewer patients leaving the ED without being seen by a physician and a shorter length of stay. ${ }^{16-20}$ However, several systematic reviews, including meta-analyses, have concluded that the evidence is not robust due to a large degree of variation in the study design and quality, intervention type and outcome measures. ${ }^{21-25}$

Interprofessional teamwork, where health workers with different professional backgrounds work together with the goal of delivering the highest quality of care, ${ }^{26}$ is an alternative approach to improving patient flow. Teamwork has been shown to improve patient safety in healthcare, though the unpredictability of the ED context poses special demands on effective team functioning and requires formal training. ${ }^{27}$ Studies of teamwork and interprofessional training have reported improvements in the quality of care, patient satisfaction and work environment, ${ }^{28-32}$ but few studies report its impact on ED throughput times. ${ }^{33}$

We describe two interventions. During the first intervention, senior physicians replaced senior nurses in the triage process. During the second intervention, the triage process was replaced by the patient being assessed and treated directly by an interprofessional team.

The aim of this study is to evaluate the impact on patient flow of three different triage processes: comprehensive nurse-led triage, senior physician-led triage and triage replaced by interprofessional teamwork. We examine patient flow in terms of ED throughput times. The research question is: can the patients' throughput times at the ED be reduced by implementing interprofessional teamwork?

\section{MATERIALS AND METHODS}

The study design was a single-centre before-and-after study. We conducted the study from May 2012 to November 2015 at the adult ED at Södersjukhuset, a 600-bed urban public teaching hospital in central Stockholm, Sweden. With 110000 annual visits, this ED is one of the largest in Scandinavia. The study material included all arrivals on weekdays from 08:00 to 21:00. We excluded patient arrivals between 21:00 to 08:00 since none of the study interventions was adopted for the night shifts. Arrivals on weekends and holidays were also excluded since the teamwork intervention was only implemented on weekdays. Each intervention was studied during a 1-year period after its implementation, with a 1-year period prior to the first intervention serving as the control period (figure 1). We

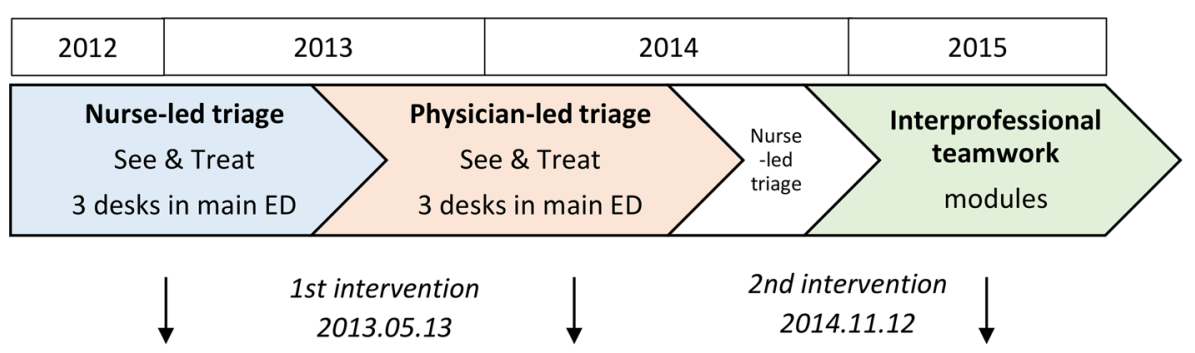
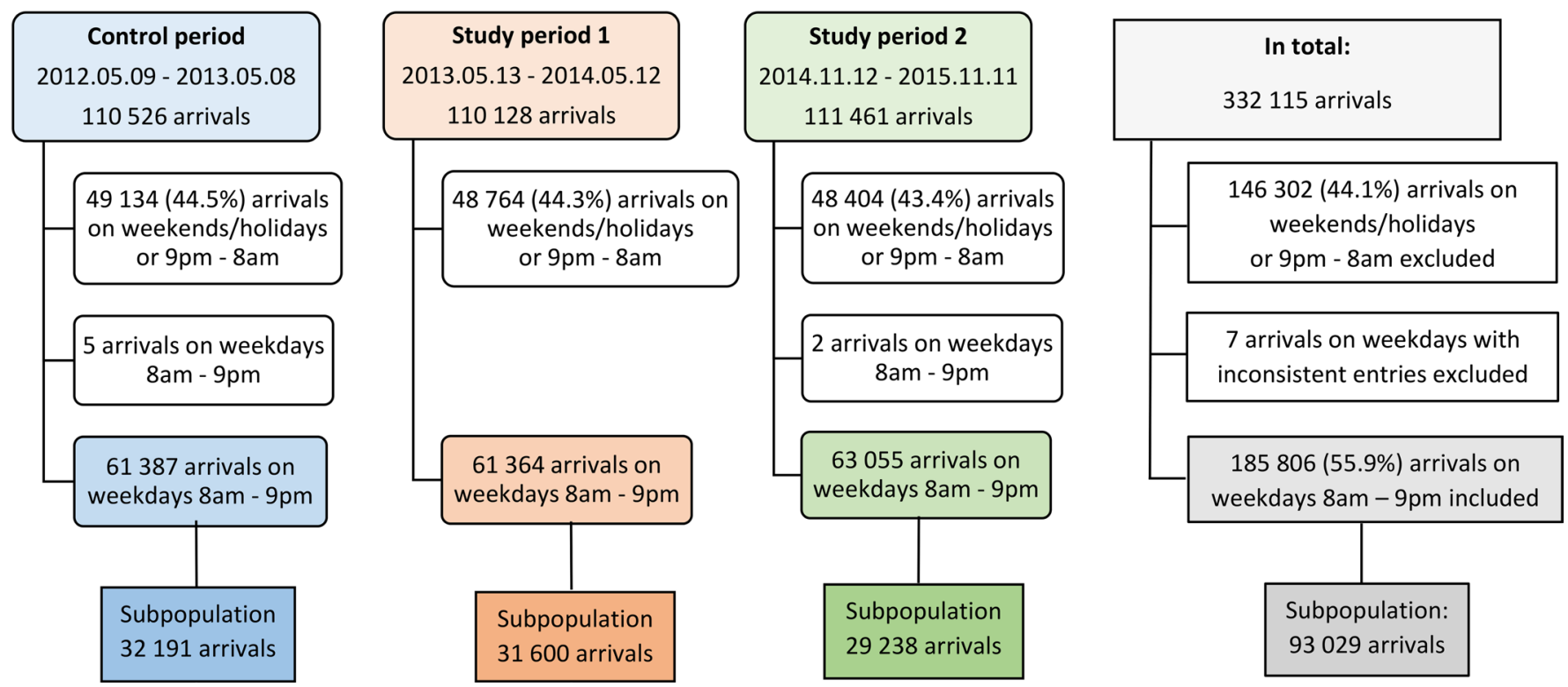

Figure 1 Timeline of the interventions and the study flow diagram. ED, emergency department. 
also excluded visits to our paediatric and gynaecology EDs because of differences in location and work processes.

\section{Nurse-led triage}

During the control period from 9 May 2012 to 8 May 2013, a comprehensive nurse-led triage process was in use. The triage teams consisted of a registered nurse and a nursing assistant who applied the Rapid Emergency Triage and Treatment System (RETTS) protocol ${ }^{34} 35$ developed in Sweden. The RETTS protocol combines the vital signs and patient history to prioritise the patients in five emergency processes according to medical urgency. For most patients, the triage nurses sent blood samples for standardised laboratory work-up. A total of eight triage team shifts were scheduled daily from 08:00 to 21:00, corresponding to 58 hours each of registered nurses and nursing assistants. During peak hours from 10:00 to 18:00, an additional registered nurse triaged the ambulance patients. A physician was available on demand by the triage nurses.

After registration, ambulant patients with minor injuries and symptoms were sent to a fast-track section, See \& Treat, while other patients were directed to the triage section unless they needed immediate care. After completing a comprehensive triage, the patient was sent to one of three desks: internal medicine, cardiology or the emergency medicine desk for surgery and orthopaedic complaints. At the desk, nursing assistants placed the patient in a room to wait for a doctor. The next available doctor assessed the patient on his or her own and left written orders for the nurses. The patient then had to wait for the next available nurse to carry out the orders, while the doctor either proceeded with documentation in a back office or took on another patient. Rooms were often occupied by patients waiting for the next step in the process. Since the work shifts started at different hours for the different professions, each doctor worked with several nurses and each nurse with several doctors during a shift.

\section{Physician-led triage}

During the first intervention from 13 May 2013 to 12 May 2014, three senior physician shifts were reassigned from each of the three desks from 08:00 to 21:00, corresponding to a total of 63 hours per day. The senior physicians formed intake teams in the triage area together with nine nursing assistant shifts, 64 hours, and two registered nurse shifts, 14 hours. Two of three cardiology intake teams included a registered nurse instead of nursing assistant, while the intake doctor of emergency medicine alternated between two rooms each staffed by a nursing assistant. Intake teams were instructed to assess all patients arriving at the ED, except those with prehospital alerts. The intake team could either discharge the patient after a brief assessment or initiate radiology and laboratory work-up and request an in-hospital bed before moving the patient to one of the three desks. The work processes at the three desks and the See \& Treat were the same as described for the nurse-led triage period.

\section{Interprofessional teamwork in modules}

During the second intervention from 12 November 2014 to 11 November 2015, interprofessional teamwork in modules was introduced on weekdays from 08:00 to 21:00. The ED facilities, including the triage area and the See \& Treat, were converted into nine modules, each equipped with two or three rooms for assessment and treatment, several bays for monitored patients and one team area. Doctors moved from the back offices so that each doctor was placed next to a nurse in the team area. A module was staffed by a flow team and two care teams. Each team consisted of a doctor and a nurse with the most senior doctor and nurse forming the flow team. An additional nursing assistant in each module helped all three teams, except in the two modules replacing See \& Treat (see figure 2). The entire staff within a module started and ended the work shift together. Four parallel modules were in operation from 08:00 to 21:00, with five additional modules added during peak hours from 10:00 to 18:00. Patients with orthopaedic and surgery complaints were streamed into separate modules, although these modules had flexibility to treat patients with other complaints when needed.

After registration, a new patient was directed to the appropriate module, where the flow nurse prioritised and re-evaluated the queuing patients with support from the flow doctor. The responsibility was transferred from the flow nurse when a care team started the assessment. The doctor and nurse in the care team collaborated to carry out the patient interview, physical examination, radiology and laboratory orders, and treatment in immediate sequence. The flow doctor supported the care teams in deciding on correct care plans for the patients.

The interventions were the results of improvement efforts made by interprofessional and multidisciplinary groups of physicians, nurses and nursing assistants. Multiple Plan-Do-Study-Act cycles $^{36}$ were carried out before the implementation of the interventions.

\section{Potential sources of bias}

We collected staffing data for each period from the work schedules for physicians and nursing staff. The scheduled working hours during weekdays from 08:00 21:00 were summed into a daily total number of hours. The ED was organised in two separate corridors. In the first corridor, physicians belonging to the departments of internal medicine and cardiology were responsible for their respective patient categories. In the second corridor, physicians belonging to the ED were responsible for all other patients in the main ED and the fast-track See \& Treat. Three different departments were thus responsible for the physician budgets and schedules, which caused staffing discrepancies. The number of working hours for physicians and nurses increased significantly in the first corridor during the study period, while it remained approximately constant in the second corridor and the See \& Treat. 


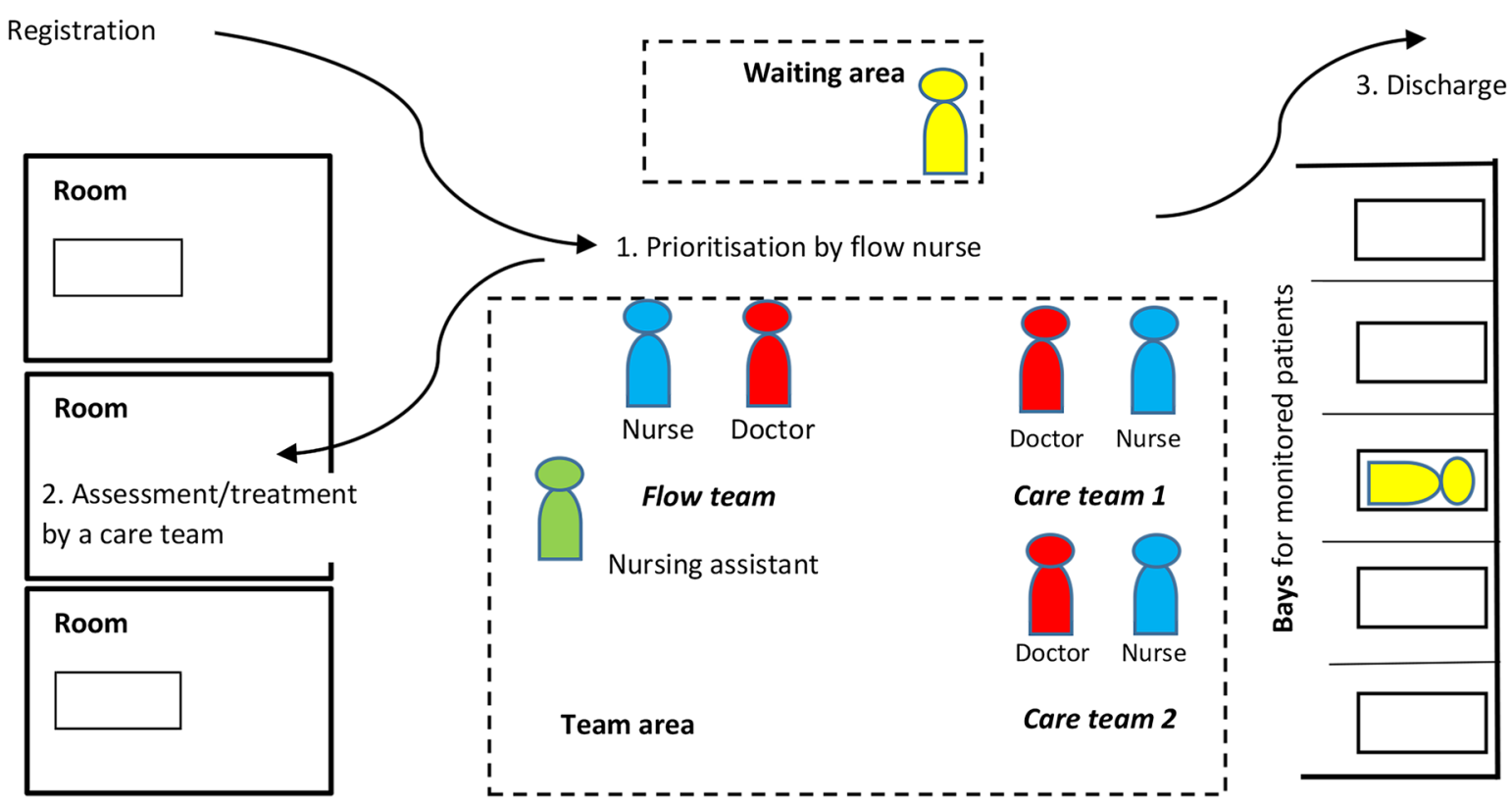

Figure 2 Interprofessional teamwork in a module: team members, work space and patient flow.

We collected registry data of available in-hospital beds and the number of admitted patients per ward weekdays at 06:00 from Belaggning.qvw, a Qlikview (QlikTech International, Radnor, Pennsylvania, USA) application used for bed occupancy reports to healthcare authorities. The daily bed occupancy rate for the wards receiving patients admitted from the adult ED was calculated as the ratio between the number of admitted patients and the available number of beds. From May 2012 to November 2015, no other process change than the studied interventions took place.

\section{Statistics}

Electronic registry data of all visits to the adult ED during the study period were extracted from the ED tracking system Akusys, after replacing patient identification numbers by unique codes. We imported the data obtained from Akusys, Belaggning.qvw and work schedules into R (The R Foundation for Statistical Computing, Vienna) for statistical analysis. We used descriptive statistics to summarise the general characteristics for each period and analysed differences between the periods using the $\chi^{2}$ test for proportions and the Mann-Whitney-Wilcoxon test for mean values.

The primary outcome measures were the total ED length of stay and the waiting time to be seen by a physician, measured from the registration time on arrival. The distributions of these variables are heavily skewed with short times for most patients, and a smaller number of very long times resulting from patients waiting for transportation or in-hospital beds. Therefore, we used the median of the time to physician and length of stay to compare the periods. We obtained $95 \%$ CIs by bootstrap simulation and calculated $\mathrm{p}$ values using Mood's test due to differences in variance between the periods. We explored the relationship between the length of stay and each individual background characteristic using scatterplots and simple linear regression. Finally, we calculated the adjusted length of stay for each 1-year period by pooling these predictors into a multivariable regression. The secondary outcome measure was the proportion of patients who left without being seen by a physician, which we analysed with the $\chi^{2}$ test. The statistical significance level was set at a two-tailed $p$ value of 0.05 for all outcomes.

\section{Patient involvement}

We did not involve patients in determining the research question and outcome measures, nor in the study design and implementation. Likewise, patients were not engaged in the interpretation and written documentation of the results. The research results may be disseminated to the study population and the relevant patient community through the local press.

\section{RESULTS}

A total of 332115 arrivals were registered during the three 1-year periods, as illustrated in the flow diagram in figure 1. The 146302 arrivals on weekends, holidays and during night shifts from 21:00 to 08:00 where the interventions were not implemented were excluded. We also excluded seven arrivals on weekdays 08:00 through 21:00 because of inconsistencies in registry entries. This meant that a total of 185806 arrivals were included. We present the population characteristics for each period in table 1 , along with mean values of in-hospital bed occupancy 
Table 1 General characteristics of the study population per 1-year period of three different triage processes

\begin{tabular}{|c|c|c|c|c|c|c|c|c|c|}
\hline Triage process & \multicolumn{3}{|c|}{ (1) Nurse-led triage } & \multicolumn{3}{|c|}{ (2) Physician-led triage } & \multicolumn{3}{|c|}{ (3) Interprofessional teamwork } \\
\hline Time period & \multicolumn{2}{|c|}{$\begin{array}{l}9 \text { May } 2012 \text { to } \\
8 \text { May } 2013\end{array}$} & \multirow{3}{*}{$\begin{array}{l}\text { Period } 1 \\
\text { versus } \\
2 \text { (P values) }\end{array}$} & \multicolumn{2}{|c|}{$\begin{array}{l}13 \text { May } 2013 \text { to } \\
12 \text { May } 2014\end{array}$} & \multirow{3}{*}{$\begin{array}{l}\text { Period } 2 \\
\text { versus } \\
3 \text { (P values) }\end{array}$} & \multicolumn{2}{|c|}{$\begin{array}{l}12 \text { November } 2014 \text { to } \\
11 \text { November } 2015\end{array}$} & \multirow{3}{*}{$\begin{array}{l}\text { Period } 3 \\
\text { versus } \\
1 \text { (P values) }\end{array}$} \\
\hline \multirow[b]{2}{*}{ Triage protocol } & \multicolumn{2}{|c|}{$\begin{array}{l}\text { Rapid Emergency } \\
\text { Triage and Treatment } \\
\text { System }\end{array}$} & & \multicolumn{2}{|l|}{ None } & & \multicolumn{2}{|l|}{ None } & \\
\hline & \multicolumn{2}{|l|}{$\mathbf{n}$} & & \multicolumn{2}{|l|}{$\mathbf{n}$} & & \multicolumn{2}{|l|}{$\mathbf{n}$} & \\
\hline Female gender & 31933 & $52.0 \%$ & 0.341 & 31706 & $51.7 \%$ & 0.354 & 32413 & $51.4 \%$ & 0.030 \\
\hline Mean age (years) & 55.3 years & SD 21.8 & 0.019 & 55.6 years & SD 21.8 & 0.009 & 56.0 years & SD 21.5 & $<0.001$ \\
\hline \multicolumn{10}{|l|}{ Arrival mode } \\
\hline Mean available beds & \multicolumn{2}{|l|}{423} & & \multicolumn{2}{|l|}{433} & & \multicolumn{2}{|l|}{408} & \\
\hline Mean bed occupancy & 391 & $92.6 \%$ & $<0.001$ & 408 & $94.3 \%$ & $<0.001$ & 398 & $97.8 \%$ & $<0.001$ \\
\hline \multicolumn{10}{|c|}{ Staffing weekdays $08: 00$ to $21: 00$} \\
\hline Physician hours & \multicolumn{2}{|l|}{249.0} & & \multicolumn{3}{|l|}{270.8} & \multicolumn{2}{|l|}{313.0} & \\
\hline $\begin{array}{l}\text { Nurse hours including } \\
\text { assistants }\end{array}$ & \multicolumn{2}{|l|}{509.8} & & \multicolumn{3}{|l|}{508.7} & \multicolumn{2}{|l|}{553.6} & \\
\hline
\end{tabular}

rate and staffing for each period. The in-hospital bed occupancy rate increased significantly during the study period, with mean occupancy rates of $92.6 \%, 94.3 \%$ and $97.8 \%$ for the respective periods.

To obtain an accurate comparison between the different triage processes, where the amount of resources available was kept as constant as possible, we also consider patients dispositioned from the emergency medicine corridor and the See \& Treat as a subgroup (table 2). In this subgroup, the total staffing per week varied over the 3-year period in an interval of $-1.5 \%$ to $+1.1 \%$ compared with a 3 -year average. Nurse staffing was constant to within $0.4 \%$ of the average, while physician staffing varied in an interval of $-4.8 \%$ to $+3.3 \%$ around the average.

A total of 93029 arrivals were dispositioned from these sections, which corresponds to $50.1 \%$ of the entire study population.

For the entire study population, the median length of stay was shortest for the teamwork period, $223 \mathrm{~min}$ compared with $226 \mathrm{~min}$ for nurse-led triage and $239 \mathrm{~min}$ for physician-led triage. The median time to physician was shortest for physician-led triage, $54 \mathrm{~min}$ compared with $66 \mathrm{~min}$ for teamwork and $98 \mathrm{~min}$ for nurse-led triage. The 95\% CIs and $p$ values are given in table 3, which shows that all differences between periods were significant with two-tailed $p$ values $<0.001$.

For the subgroup, the median length of stay was shortest for the teamwork period, $228 \mathrm{~min}$ compared with $232 \mathrm{~min}$ for nurse-led triage and $250 \mathrm{~min}$ for physician-led triage. The median time to physician was shortest for physician-led triage, $56 \mathrm{~min}$ compared with $74 \mathrm{~min}$ for teamwork and $116 \mathrm{~min}$ for nurse-led triage. The $95 \%$ CIs and $p$ values are listed in table 4 , which shows that all differences between periods were significant with two-tailed $\mathrm{p}$ values $<0.01$. Table 4 also shows that the differences in outcome measures were similar both for discharged and admitted patients. The length-of-stay distribution for each study period is shown in figure 3 and the distribution of the time to physician in figure 4 . Both distributions are heavily skewed. The asymmetry of the length-of-stay distribution increased from period to period, with a skewness of 1.35 for nurse-led triage, 1.46 for physician-led triage and 1.55 for teamwork.

After pooling data from all three periods, we explored each population characteristic as a predictor of the length of stay using simple linear regression analysis. The resulting estimate indicates a length of stay which is 64 min longer for patients over 74 years, 20 min longer for female patients, $80 \mathrm{~min}$ longer for ambulance patients and $68 \mathrm{~min}$ shorter for ambulance patients arriving with prehospital alert. We have chosen these arrival modes as more reliable indicators of patient severity since triage severity was registered in different ways in the different intervention periods. The length-of-stay estimate increased by $0.6 \mathrm{~min}$ with each unit increase in daily arrival volume. The observed increase of the inpatient bed occupancy rate from $92.6 \%$ for nurse-led triage to $97.8 \%$ for teamwork was estimated to increase the length of stay by $11 \mathrm{~min}$. Finally, we pooled all these explored predictors in a multivariable regression analysis and found that the 
Table 2 General characteristics of the subgroup with approximately constant staffing resources for the different triage processes

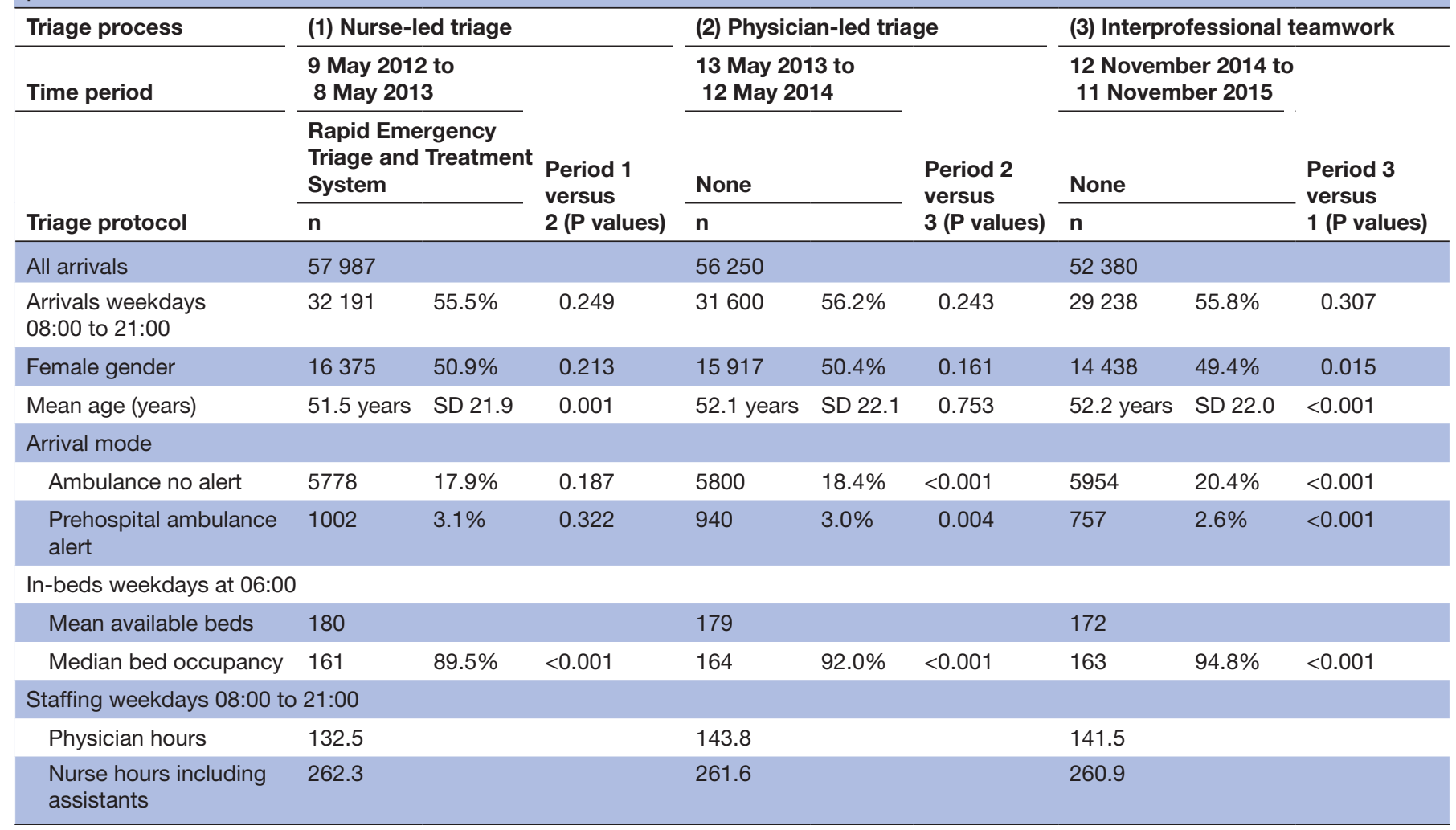

adjusted length-of-stay estimate for teamwork was $12 \mathrm{~min}$ shorter than for nurse-led triage and 21 min shorter than for physician-led triage. We have listed the estimates with SEs and $p$ values from the simple and multivariable regression analyses in table 5 .

We also conducted regression analyses of the subgroup and present the estimates in table 6, including SEs and $\mathrm{p}$ values. In the final multivariable regression analysis, we found that the adjusted length-of-stay estimate for teamwork was $16 \mathrm{~min}$ shorter than for nurse-led triage and 23 min shorter than for physician-led triage.

In the subgroup, the proportion of patients who left without being seen by a physician was smallest for physician-led triage, $1.2 \%$ compared with $1.9 \%$ for nurse-led triage and $3.2 \%$ for teamwork (table 4). The corresponding rate of the entire study population was also lowest for physician-led triage and highest for teamwork period (table 3). All differences were of statistical significance.

\section{DISCUSSION}

This study evaluated the impacts on patient flow of three different triage processes in terms of ED throughput times: nurse-led triage, senior physician-led triage and interprofessional teamwork. The main finding was the shortest median length of stay observed for the teamwork period. Another main finding was the longest length of stay observed for physician-led triage, despite the shortest time to physician for this period.
In the multivariable regression analysis, staffing was not included as an independent variable. This is due to the structure of the staffing data, where schedules were constant in the teamwork period, and only a single minor adjustment was made in each of the other periods.

This results in a very high degree of correlation between the staffing and triage period variables, which causes a collinearity problem when including both variables in the regression. ${ }^{37}$ The restriction to a subgroup of approximately constant resources was introduced to provide a more accurate comparison of working processes in this situation.

Interprofessional teamwork is based on the following principles, which we believe contribute to the increased efficiency found in this study: reducing the number of patients each staff member is responsible for, reducing the number of staff members encountered by the patient, deciding appropriate treatment plans from the start and carrying out the plans immediately. For this to happen, work shifts started and ended at the same time, and roles and responsibilities were clearly defined for all members in a module. Each module had its own fully equipped rooms and team area, thus creating smaller subsets within the large ED to enhance interprofessional teamwork. This may be particularly relevant to large EDs since a correlation has been found between longer length of stay and increasing annual ED volumes. ${ }^{38}$ Welch $e t a l^{38}$ suggested reducing the volume of a large ED by creating smaller subsets or clinical microsystems as an approach to 


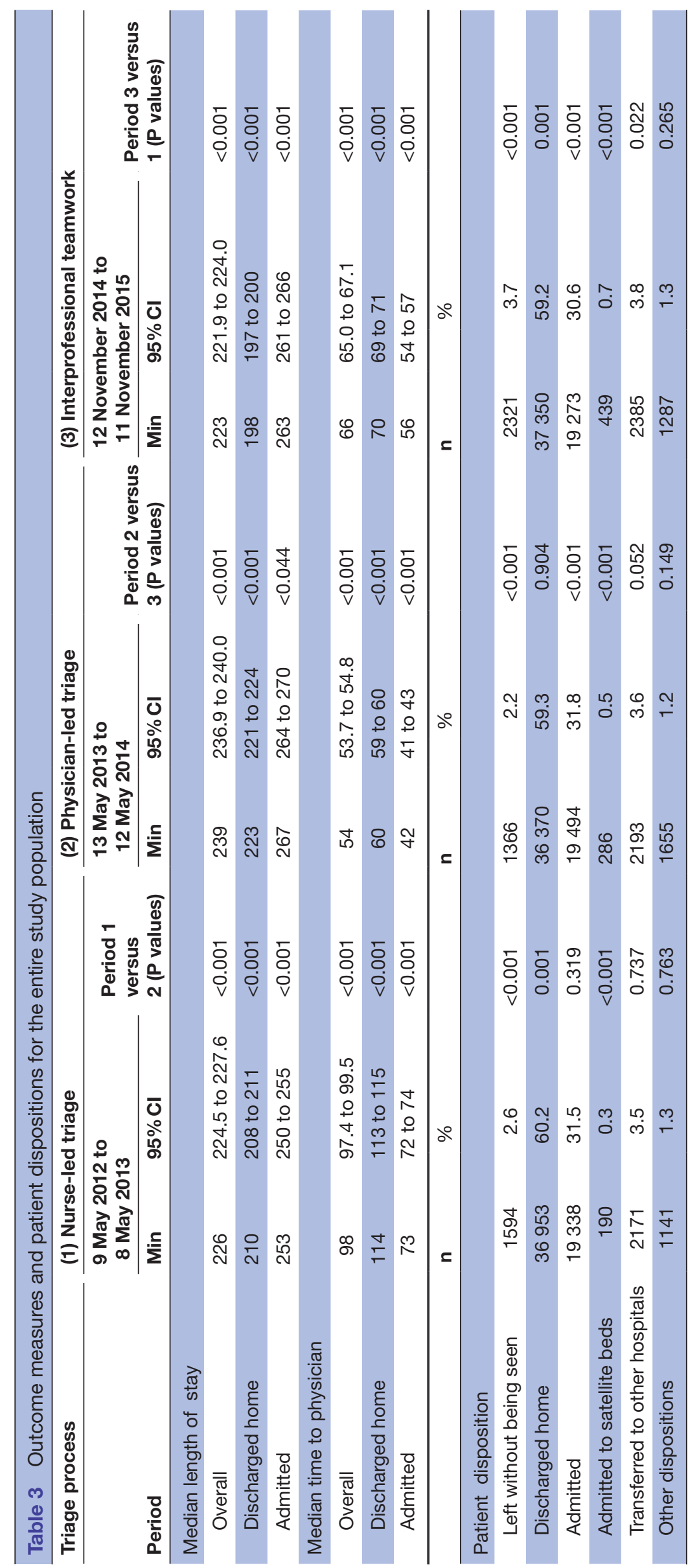

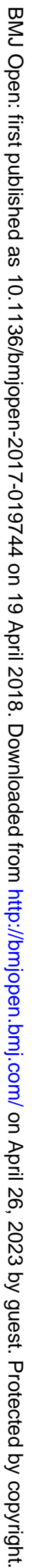




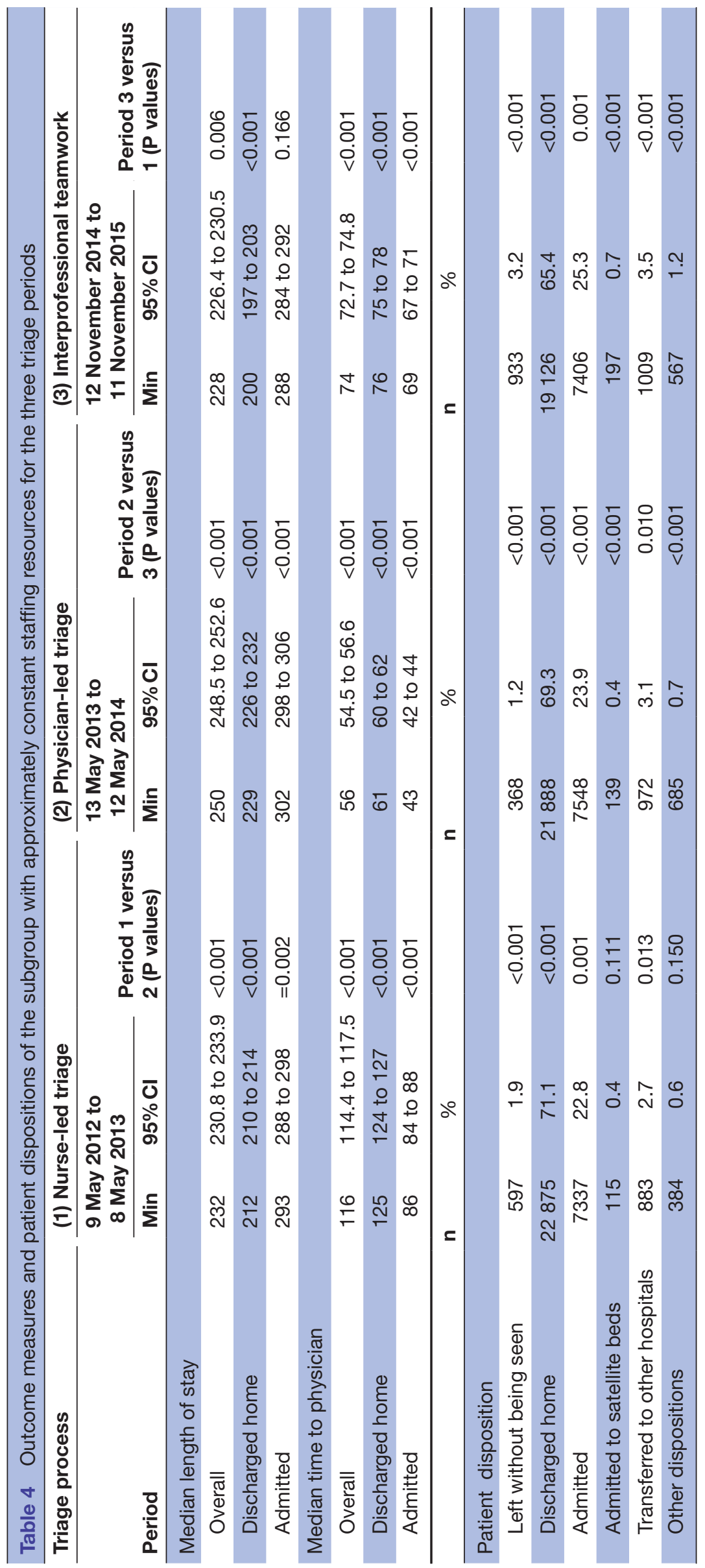

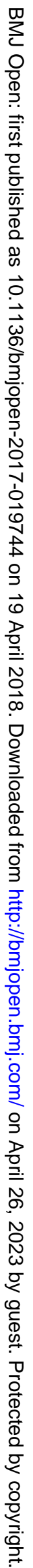




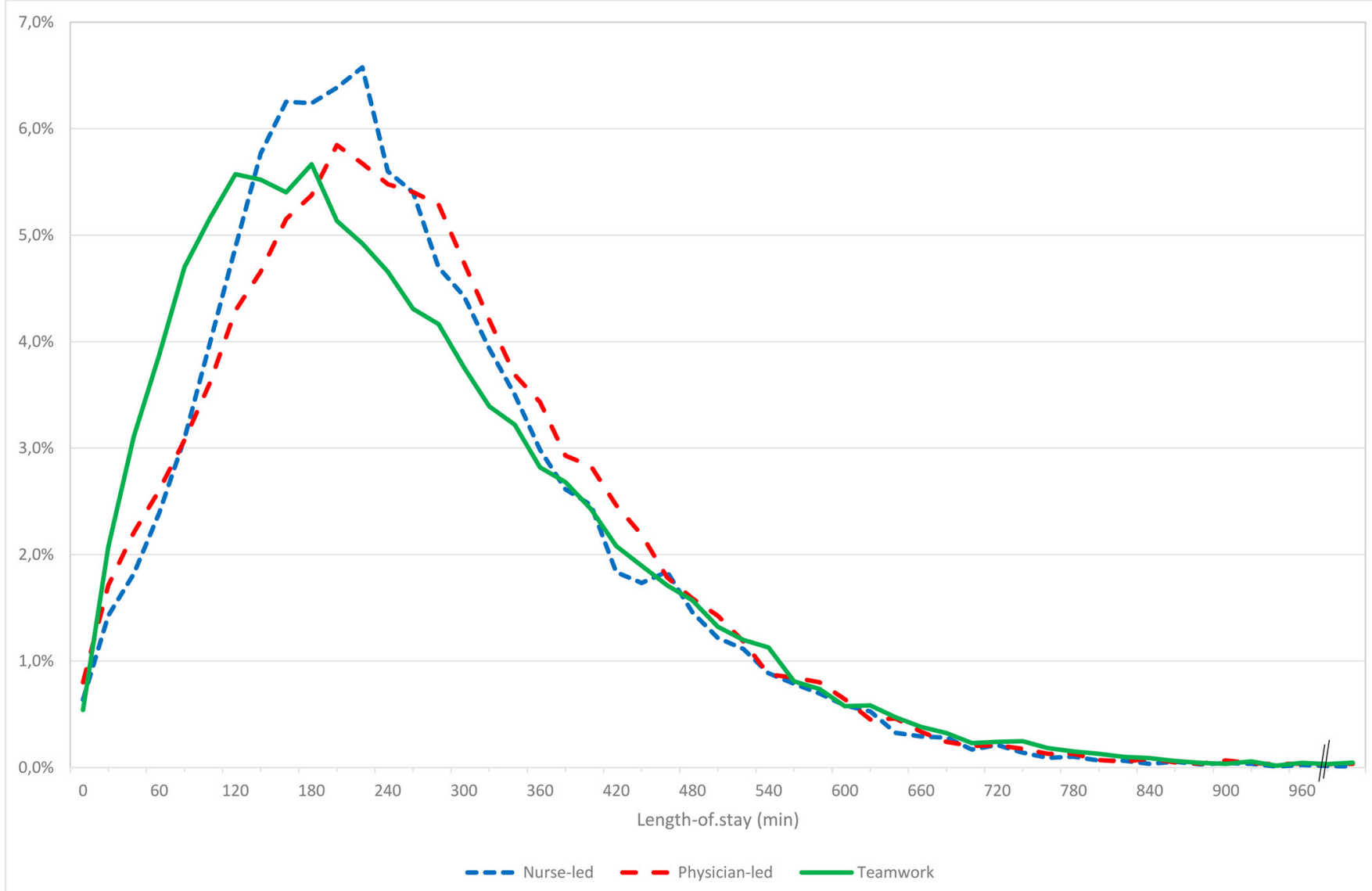

Figure 3 Length-of-stay distribution per triage period of the subpopulation.

improve the efficiency. Improvement in communication and patient safety, ${ }^{29}{ }^{31}$ staff $^{40}$ and patient satisfaction ${ }^{28} 30$ are documented effects of interprofessional teamwork. The present study shows that teamwork can also improve ED throughput times. To the best of our knowledge, only one previous study has reported a small but significant reduction of the length of stay in the case of physiciannurse teamwork. ${ }^{33}$

One may note that a smaller proportion of patients in the subpopulation studied were discharged home during the teamwork period, $65.4 \%$ compared with $71.1 \%$ for nurse-led triage and $69.3 \%$ for physician-led triage. This may be due to the fast-track See \& Treat having been replaced by two modules for ambulant patients, one in each ED corridor. Internal medicine complaints previously treated at See \& Treat were transferred to modules in the other corridor. The median length of stay was shorter for patients discharged home than those admitted for all periods, $88 \mathrm{~min}$ shorter for teamwork, $73 \mathrm{~min}$ for physician-led triage and $81 \mathrm{~min}$ for nurse-led triage (table 4). This observed shift towards more serious complaints could be interpreted as providing further support for a higher efficiency of the teamwork process. Another observation supporting this view was the increasing skewness of the length-of-stay distribution from period to period, which implies an increasing proportion of patients with a short length of stay in the presence of a smaller number of patients with increasing length of stay. This may have been caused by the increasing inpatient bed occupancy from period to period.

When senior physicians replaced nurses in triage in the first intervention, the median time to physician decreased by $60 \mathrm{~min}$. In a meta-analysis, Abdulwahid et a $t^{25}$ estimated a reduction by $26 \mathrm{~min}$ from two randomised controlled trials (RCTs) ${ }^{41} 42$ and 15 min from nine non-RCTs. Our first intervention increased the median length of stay by $18 \mathrm{~min}$, in contrast to the estimated reduction by $29 \mathrm{~min}$ of the meta-analysis. Four of the publications included in the meta-analysis appear to report different follow-up lengths of an identical intervention in the same $\mathrm{ED},{ }^{1943-45}$ which may overestimate the effect size. Most studies reporting reduced length of stay introduced additional physicians in the triage interventions, ${ }^{16} 1719$ while in the first intervention of this study the senior physicians were reassigned to the triage. This may explain the increased length of stay in our study. However, Choi et al reduced the waiting time and processing time by reassigning a senior physician to the triage process. ${ }^{18}$ To our knowledge, two studies found no significant changes in length of stay, ${ }^{46}$ while one study has reported a significant $15 \mathrm{~min}$ increase along with an $11 \%$ increase of orders for diagnostic radiology. ${ }^{48}$ For patients dispositioned by a second physician at the main ED after senior physician assessment at triage, Traub et al found a 25 min longer length of stay. ${ }^{20}$ 


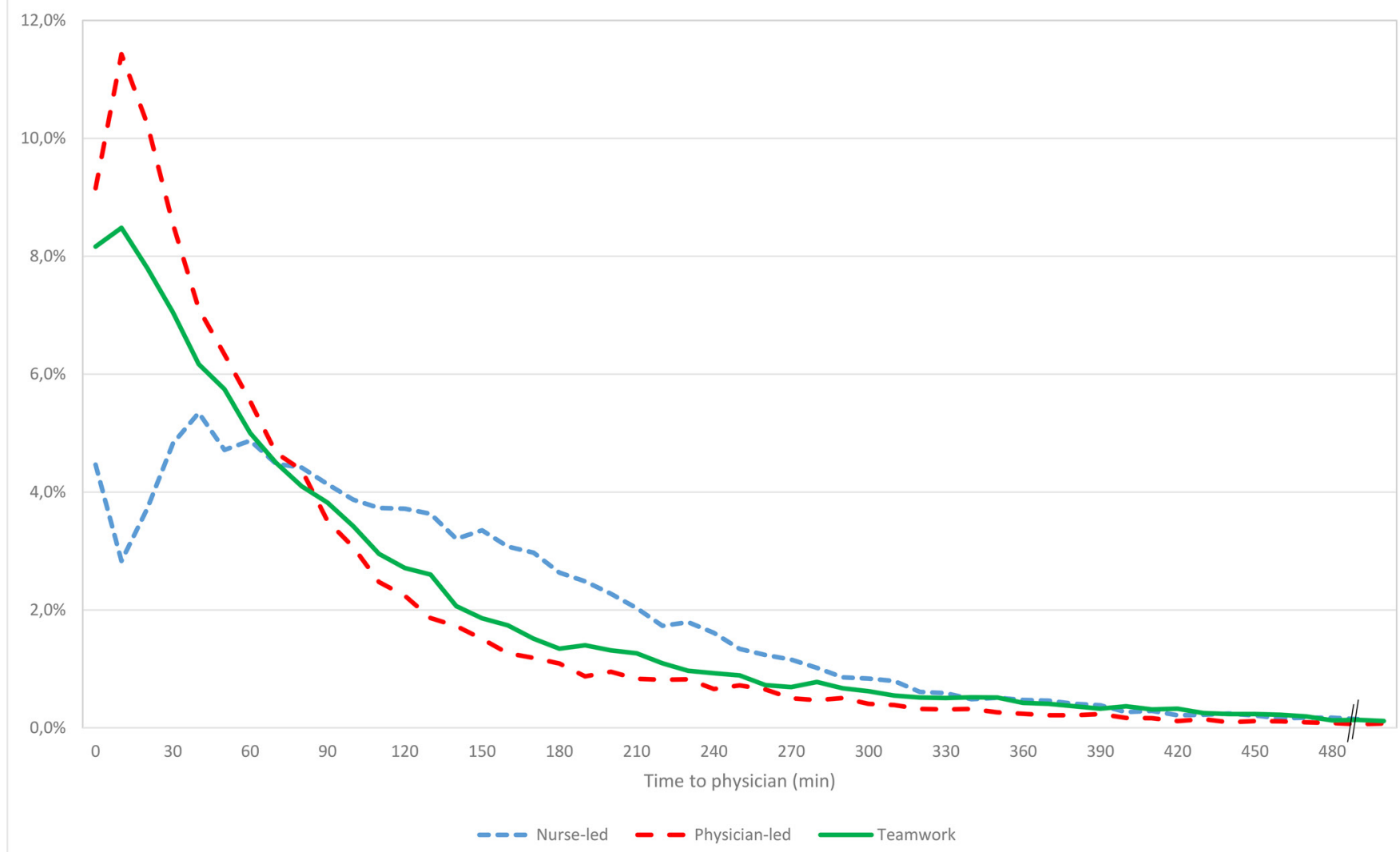

Figure 4 Time to physician distribution per triage period of the subpopulation.

Although Choi et al found significant reductions of the time to physician and length of stay, they also described 'stressful, pressurised and risky' working conditions for the senior physician in triage. ${ }^{18}$ When Burström et al compared three EDs with different triage processes, they found the shortest length of stay for senior physician-led triage ${ }^{49}$ However, this ED also applied interprofessional teamwork. The senior physician at triage planned the patients' ED stay and communicated the plan to teams consisting of a junior physician and a nurse who worked in parallel. At the other two EDs, physicians and nurses worked separately and sequentially.

The smallest proportion of patients who left without being seen by a physician was observed for physician-led triage, which is in line with the significant decrease reported by previous studies of physicians at triage. ${ }^{1645}$ We observed the highest rate for the teamwork period, despite a 46 min shorter time to physician compared with

Table 5 Regression analysis of the entire study population: predictors of length of stay explored individually by linear regression and pooled in multivariable regression

\begin{tabular}{|c|c|c|c|c|c|c|}
\hline & \multicolumn{3}{|c|}{ Simple regression } & \multicolumn{3}{|c|}{ Multivariable regression } \\
\hline & Estimate & SE & $P$ values & Estimate & SE & $P$ values \\
\hline Age (per year) & 1.42 & 0.02 & $<0.001$ & 1.12 & 0.02 & $<0.001$ \\
\hline Gender female (yes/no) & 19.90 & 0.78 & $<0.001$ & 14.69 & 0.75 & $<0.001$ \\
\hline \multicolumn{7}{|l|}{ Arrival mode } \\
\hline Ambulance without alert (yes/no) & 79.92 & 0.89 & $<0.001$ & 58.25 & 0.94 & $<0.001$ \\
\hline Ambulance with prehospital alert (yes/no) & -68.02 & 1.78 & $<0.001$ & -64.34 & 1.77 & $<0.001$ \\
\hline Daily occupancy rate at 06:00 (0-1) & 217.42 & 7.39 & $<0.001$ & 233.44 & 7.87 & $<0.001$ \\
\hline Daily total arrival volume & 0.60 & 0.01 & $<0.001$ & 0.69 & 0.01 & $<0.001$ \\
\hline \multicolumn{7}{|l|}{ Difference compared with teamwork period } \\
\hline Nurse-led triage period (yes/no) & & & & 12.07 & 1.01 & $<0.001$ \\
\hline Physician-led triage period (yes/no) & & & & 20.91 & 0.96 & $<0.001$ \\
\hline
\end{tabular}


Table 6 Regression analysis of the subgroup: predictors of length of stay explored individually by linear regression and pooled in multivariable regression

\begin{tabular}{|c|c|c|c|c|c|c|}
\hline & \multicolumn{3}{|c|}{ Simple regression } & \multicolumn{3}{|c|}{ Multivariable regression } \\
\hline & Estimate & SE & $P$ values & Estimate & SE & $P$ values \\
\hline Age (per year) & 1.73 & 0.03 & $<0.001$ & 1.19 & 0.02 & $<0.001$ \\
\hline Gender female (yes/no) & 26.70 & 1.07 & $<0.001$ & 17.84 & 1.03 & $<0.001$ \\
\hline \multicolumn{7}{|l|}{ Arrival mode } \\
\hline Ambulance without alert (yes/no) & 108.38 & 1.33 & $<0.001$ & 84.26 & 1.40 & $<0.001$ \\
\hline Ambulance with prehospital alert (yes/no) & -37.85 & 3.19 & $<0.001$ & -28.65 & 3.09 & $<0.001$ \\
\hline Daily in-bed occupancy at 06:00 (0-1) & 208.23 & 10.26 & $<0.001$ & 224.44 & 10.76 & $<0.001$ \\
\hline Daily total arrival volume & 0.61 & 0.02 & $<0.001$ & 0.70 & 0.02 & $<0.001$ \\
\hline \multicolumn{7}{|l|}{ Difference compared with teamwork period } \\
\hline Nurse-led triage period (yes/no) & & & & 15.56 & 1.39 & $<0.001$ \\
\hline Physician-led triage period (yes/no) & & & & 23.46 & 1.33 & $<0.001$ \\
\hline
\end{tabular}

nurse-led triage. Although this rate is often used as an indicator of crowding, patients who leave without being seen by a physician have been shown to be at a lower risk of death or admission within 7 days compared with patients who were seen by physicians and discharged home. ${ }^{50}$ These authors found no association between EDs with high annual left without being seen rate and risk of death or admission. Nonetheless, the higher rate for the teamwork process calls for further exploration and should be addressed.

\section{Strengths and limitations}

The main strength of this study is the large population which enables the evaluation of the process rather than the performance of individual doctors or nurses. Another strength is the control and study periods of 1 year each, which compensates for seasonal fluctuations and allows each intervention to stabilise after the initial implementation. We were only able to identify one other study of a similar population size and length of time ${ }^{19}$ Furthermore, analyses of multiple interventions and studies comparing several triage processes are rare.

This study has several limitations. First, this is a singlecentre study in one large, busy urban ED and the results may not be generalisable to other ED settings. EDs differ from each other in aspects of input, throughput and output. ${ }^{51}$ In addition, before-and-after studies may not claim a causality between the intervention and the outcomes, although we have chosen the periods with no other simultaneous process changes. We did not include patients who arrived during night shifts or on weekends and holidays since the interventions did not include these work shifts. However, we analysed outcome measures for all patients arriving before 21:00, including those treated by the night shift. The second intervention was a deeper redesign of the entire ED to enable a new approach to the triage process. Finally, a limitation shared by previous studies is the use of throughput times as surrogate outcome measures for ED quality and patient safety.
However, the outcome measures we chose have been shown to be indicators of patient outcome $e^{50} 53$ and patient satisfaction. $^{545}$

Future studies of interprofessional teamwork in EDs with a multicentre design are of value to confirm our findings, as well as studies with cost-effectiveness evaluations.

\section{CONCLUSION}

The median length of stay was shortest for interprofessional teamwork in modules. It was longest for physician-led triage, despite the shortest time to physician of all studied periods. Interprofessional teamwork in modules may be an interesting approach to improve timeliness in large busy EDs.

Acknowledgements Mats Nordahl provided statistical advice. Bo Herrlin facilitated the improvement groups, and members of these groups designed the interventions by repeated test cycles before they were implemented.

Contributors All authors participated in the initiation and development of the study design. JL performed the literature search with inputs from IM, SP and NF. JL collected and analysed the data and wrote the initial draft. IM, SP and NF participated in critical revisions and have approved the final manuscript. The guarantor is $\mathrm{JL}$.

Funding Stockholm County Council sponsored the study through research and development funds dedicated for the Department of Emergency Care at Södersjukhuset and through grants dedicated for work environment improvement.

Disclaimer The funder had no input to the study design, data collection or interpretation, writing of report or submission for publication.

Competing interests None declared.

Patient consent Not required.

Ethics approval The study was approved by the Regional Ethical Review Board of Stockholm, ref. no. 2016/109-31/5. I

Provenance and peer review Not commissioned; externally peer reviewed.

Data sharing statement Full dataset and statistical codes are available from the corresponding author.

Open Access This is an Open Access article distributed in accordance with the Creative Commons Attribution Non Commercial (CC BY-NC 4.0) license, which permits others to distribute, remix, adapt, build upon this work non-commercially, and license their derivative works on different terms, provided the original work is 
properly cited and the use is non-commercial. See: http://creativecommons.org/ licenses/by-nc/4.0/

(C) Article author(s) (or their employer(s) unless otherwise stated in the text of the article) 2018. All rights reserved. No commercial use is permitted unless otherwise expressly granted.

\section{REFERENCES}

1. Derlet RW, Richards JR. Overcrowding in the nation's emergency departments: complex causes and disturbing effects. Ann Emerg Med 2000;35:63-8.

2. Hoot NR, Aronsky D. Systematic review of emergency department crowding: causes, effects, and solutions. Ann Emerg Med 2008;52:126-36.

3. Pines JM, Hilton JA, Weber EJ, et al. International perspectives on emergency department crowding. Acad Emerg Med 2011;18:1358-70.

4. The College of Emergency Medicine. Crowding in emergency departments. 2014 https://secure.rcem.ac.uk/code/document.asp? ID=6296 (accessed 11 Jul 2017).

5. American College of Emergency Physicians. Emergency department crowding: high impact solutions. 2016 https://www.acep.org/ Clinical-Practice-Management/Emergency-Medicine-Crowding-andBoarding/ (accessed 12 Jul 2017).

6. Higginson I. Emergency department crowding. Emerg Med J 2012;29:437-43.

7. Bernstein SL, Aronsky D, Duseja R, et al. The effect of emergency department crowding on clinically oriented outcomes. Acad Emerg Med 2009;16:1-10.

8. Carter EJ, Pouch SM, Larson EL. The relationship between emergency department crowding and patient outcomes: a systematic review. J Nurs Scholarsh 2014;46:106-15.

9. Jenkins MG, Rocke LG, McNicholl BP, et al. Violence and verbal abuse against staff in accident and emergency departments: a survey of consultants in the UK and the Republic of Ireland. J Accid Emerg Med 1998;15:262-5.

10. Rondeau KV, Francescutti LH. Emergency department overcrowding: the impact of resource scarcity on physician job satisfaction. $J$ Healthc Manag 2005;50:327-41.

11. Healy S, Tyrrell M. Stress in emergency departments: experiences of nurses and doctors. Emerg Nurse 2011;19:31-7.

12. Gilboy N, Travers D, Wuerz R. Re-evaluating triage in the new millennium: A comprehensive look at the need for standardization and quality. J Emerg Nurs 1999;25:468-73.

13. Iserson KV, Moskop JC. Triage in medicine, part I: Concept, history, and types. Ann Emerg Med 2007;49:275-81.

14. Farrohknia N, Castrén M, Ehrenberg A, et al. Emergency department triage scales and their components: a systematic review of the scientific evidence. Scand J Trauma Resusc Emerg Med 2011;19:42.

15. Robinson DJ. An integrative review: triage protocols and the effect on ED length of stay. J Emerg Nurs 2013;39:398-408.

16. Partovi SN, Nelson BK, Bryan ED, et al. Faculty triage shortens emergency department length of stay. Acad Emerg Med 2001;8:990-5.

17. Holroyd BR, Bullard MJ, Latoszek $\mathrm{K}$, et al. Impact of a triage liaison physician on emergency department overcrowding and throughput: a randomized controlled trial. Acad Emerg Med 2007;14:702-8.

18. Choi YF, Wong TW, Lau CC. Triage rapid initial assessment by doctor (TRIAD) improves waiting time and processing time of the emergency department. Emerg Med J 2006;23:262-5.

19. Rogg JG, White BA, Biddinger PD, et al. A long-term analysis of physician triage screening in the emergency department. Acad Emerg Med 2013;20:374-80.

20. Traub SJ, Wood JP, Kelley J, et al. Emergency department rapid medical assessment: overall effect and mechanistic considerations. $J$ Emerg Med 2015;48:620-7.

21. Rowe BH, Guo X, Villa-Roel C, et al. The role of triage liaison physicians on mitigating overcrowding in emergency departments: a systematic review. Acad Emerg Med 2011;18:111-20.

22. Oredsson $\mathrm{S}$, Jonsson $\mathrm{H}$, Rognes J, et al. A systematic review of triage-related interventions to improve patient flow in emergency departments. Scand J Trauma Resusc Emerg Med 2011;19:43.

23. Elder E, Johnston AN, Crilly J. Review article: systematic review of three key strategies designed to improve patient flow through the emergency department. Emerg Med Australas 2015;27:394-404.

24. Ming T, Lai A, Lau PM. Can Team Triage Improve Patient Flow in the Emergency Department? A Systematic Review and Meta-Analysis. Adv Emerg Nurs J 2016;38:233-50.
25. Abdulwahid MA, Booth A, Kuczawski M, et al. The impact of senior doctor assessment at triage on emergency department performance measures: systematic review and meta-analysis of comparative studies. Emerg Med J 2016;33:504-13.

26. World Health Organization. Framework for action on interprofessional education \& collaborative practice. Geneva: World Health Organization, 2010. http://apps.who.int/iris/bitstream/10665/70185/ 1/WHO_HRH_HPN_10.3_eng.pdf (accessed 4 Sep 2017).

27. Fernandez R, Kozlowski SW, Shapiro MJ, et al. Toward a definition of teamwork in emergency medicine. Acad Emerg Med 2008;15:1104-12.

28. Debehnke D, Decker MC. The effects of a physician-nurse patient care team on patient satisfaction in an academic ED. Am J Emerg Med 2002;20:267-70.

29. Morey JC, Simon R, Jay GD, et al. Error reduction and performance improvement in the emergency department through formal teamwork training: evaluation results of the MedTeams project. Health Serv Res 2002;37:1553-81.

30. Patel PB, Vinson DR. Team assignment system: expediting emergency department care. Ann Emerg Med 2005;46:499-506.

31. Epstein NE. Multidisciplinary in-hospital teams improve patient outcomes: A review. Surg Neurol Int 2014;5(Suppl 7):295-303.

32. Fung L, Boet S, Bould MD, et al. Impact of crisis resource management simulation-based training for interprofessional and interdisciplinary teams: A systematic review. J Interprof Care 2015;29:433-44.

33. Muntlin Athlin A, von Thiele Schwarz U, Farrohknia N. Effects of multidisciplinary teamwork on lead times and patient flow in the emergency department: a longitudinal interventional cohort study. Scand J Trauma Resusc Emerg Med 2013;21:76.

34. Widgren BR, Jourak M. Medical Emergency Triage and Treatment System (METTS): a new protocol in primary triage and secondary priority decision in emergency medicine. J Emerg Med 2011;40:623-8.

35. Westergren H, Ferm M, Häggström P. First evaluation of the paediatric version of the Swedish rapid emergency triage and treatment system shows good reliability. Acta Paediatr 2014;103:305-8.

36. Moen R. Foundation and history of the PDSA Cycle: The W Edwards Deming Institute;. 2016 https://deming.org/uploads/paper/PDSA History_Ron_Moen.pdf (accessed 1 Jul 2017).

37. Belsley DA, Kuh E, Welsch RE. Regression Diagnostics: Identifying Influential Data and Sources of Collinearity. New York: Wiley, 1980.

38. Welch SJ, Augustine JJ, Dong L, et al. Volume-related differences in emergency department performance. Jt Comm J Qual Patient Saf 2012;38:395-AP1.

39. Handel DA, Sun B, Augustine JJ, et al. Association among Emergency Department Volume Changes, Length of Stay, and Leaving Before Treatment Complete. Hosp Top 2015;93:53-9.

40. Ajeigbe DO, McNeese-Smith D, Leach LS, et al. Nurse-physician teamwork in the emergency department: impact on perceptions of job environment, autonomy, and control over practice. J Nurs Adm 2013;43:142-8.

41. Subash F, Dunn F, McNicholl B, et al. Team triage improves emergency department efficiency. Emerg Med J 2004;21:542-4.

42. Cheng I, Lee J, Mittmann N, et al. Implementing wait-time reductions under Ontario government benchmarks (Pay-for-Results): a Cluster Randomized Trial of the Effect of a Physician-Nurse Supplementary Triage Assistance team (MDRNSTAT) on emergency department patient wait times. BMC Emerg Med 2013;13:17.

43. White BA, Brown DF, Sinclair J, et al. Supplemented Triage and Rapid Treatment (START) improves performance measures in the emergency department. J Emerg Med 2012;42:322-8.

44. Soremekun OA, Biddinger PD, White BA, et al. Operational and financial impact of physician screening in the ED. Am J Emerg Med 2012;30:532-9.

45. Soremekun OA, Capp R, Biddinger PD, et al. Impact of physician screening in the emergency department on patient flow. $J$ Emerg Med 2012;43:509-15.

46. Davis RA, Dinh MM, Bein KJ, et al. Senior work-up assessment and treatment team in an emergency department: a randomised control trial. Emerg Med Australas 2014;26:343-9.

47. French S, Lindo JL, Williams Jean EW, et al. Doctor at triage - effect on waiting time and patient satisfaction in a Jamaican hospital. Int Emerg Nurs 2014;22:123-6.

48. Lauks J, Mramor B, Baumgartl K, et al. Medical Team Evaluation: Effect on Emergency Department Waiting Time and Length of Stay. PLoS One 2016;11:e0154372.

49. Burström L, Nordberg M, Ornung G, et al. Physician-led team triage based on lean principles may be superior for efficiency and quality? A comparison of three emergency departments with different triage models. Scand J Trauma Resusc Emerg Med 2012;20:57. 
50. Guttmann A, Schull MJ, Vermeulen MJ, et al. Association between waiting times and short term mortality and hospital admission after departure from emergency department: population based cohort study from Ontario, Canada. BMJ 2011;342:d2983.

51. Asplin BR, Magid DJ, Rhodes KV, et al. A conceptual model of emergency department crowding. Ann Emerg Med 2003;42:173-80.

52. Sprivulis PC, Da Silva JA, Jacobs IG, et al. The association between hospital overcrowding and mortality among patients admitted via Western Australian emergency departments. Med J Aust 2006;184:208-12.
53. Ackroyd-Stolarz S, Read Guernsey J, Mackinnon NJ, et al. The association between a prolonged stay in the emergency department and adverse events in older patients admitted to hospital: a retrospective cohort study. BMJ Qual Saf 2011;20:564-9.

54. Pines JM, lyer S, Disbot M, et al. The effect of emergency department crowding on patient satisfaction for admitted patients. Acad Emerg Med 2008:15:825-31.

55. Pines JM, Garson C, Baxt WG, et al. ED crowding is associated with variable perceptions of care compromise. Acad Emerg Med 2007;14:1176-81. 